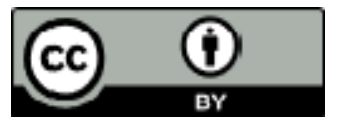

\section{Cristiano Broccias}

University of Genoa
UDC 81'367.6=111

Original scientific article

Accepted for publication on 26.07. 2021

https://doi.org/10.29162/jez.2021.8

\title{
A new look at word classes in Cognitive Grammar
}

\begin{abstract}
Langacker's Cognitive Grammar aims to offer a conceptual characterization of word classes. Pivotal to this endeavor is the distinction between a "thing" and a "relation" as well as the claim that relations can be scanned either sequentially or summarily. The difference between the two scanning modes is essential to separate, for example, enter as a finite verb from the dynamic preposition into. In this paper, the debate on the status and relevance of the two scanning modes is revisited and the very notion of relation is subjected to close scrutiny, thus revealing its potentially problematic nature. A new way of looking at word classes based on the notions of evolving vs. non-evolving categorization sequences, extrinsicality vs. intrinsicality, and anchorability is developed, which constitutes a radical departure from Cognitive Grammar despite being compatible with its fundamental assumptions.
\end{abstract}

Key words: word classes; Cognitive Grammar; summary scanning; sequential scanning; relation.

\section{Introduction}

Langacker's Cognitive Grammar (CG), see Langacker (1987, 1990, 1991, 1999, 2008a, 2009; Taylor 2002), aims to offer a conceptual characterisation of word classes. As will be shown in Section 2, this characterisation rests on two ingredients. One is the distinction between a "thing" and a "relation(ship)". A thing, a technical term in CG, is any product of our cognitive abilities for grouping and reification. A relation, instead, profiles interconnections. The other key element for word class identification is the difference drawn between two modes of temporal scanning of relations. CG claims that relations can be scanned either sequentially, as in a motion picture, or summarily, as in a multiple exposure photo. The difference between the two scanning modes is regarded as crucial to distinguishing, for 


\section{Cristiano Broccias: \\ A new look at word classes in Cognitive Grammar}

example, the relation profiled by the verb enter, which is said to involve sequential scanning, and the relation profiled by the "dynamic" preposition into, which is said to involve summary scanning.

Nevertheless, the status and relevance of sequential and summary scanning to word class classification has been called into question, see Broccias and Hollmann (2007) but also Langacker (2008b) for possible counterarguments. Thus, Section 3 will revisit this debate but will also provide some new discussion of the notion of "relation", which, too, is shown to be potentially problematic. The paper then proposes in Section 4 a new way of looking at Langacker's relations which is still consistent with CG assumptions but which leads to a significant restructuring of the CG description of word classes.

\section{The CG account of word classes}

In CG all linguistic expressions are bipolar. In other words, a given expression is said to be made up of a semantic pole, corresponding to the expression's meaning (broadly construed), and a phonological pole, describing how the given expression is pronounced. For example, the semantic pole of the noun train, abbreviated as [TRAIN], specifies, among other things, that this expression depicts a means of transport consisting of (usually) more than one carriage pulled by an engine along a railway line, while its phonological pole consists, in British English and American English, of the sequence of segments [treIn]. ${ }^{1}$ The two poles are connected to each other or, technically speaking, symbolize each other and thus make up a symbolic assembly abbreviated as [[TRAIN]/[treIn]], which thus comprises the two poles as well as the symbolizing relation existing between them. ${ }^{2}$

Crucially, CG claims that this bipolar characterisation is not exclusive to single words but is also valid for expressions "smaller" than words such as bound morphemes and "larger" expressions such as syntactic constructions. For example, the bound morpheme -er, as in driver, trainer, smoker, etc., is analysable as an assembly of a semantic pole that typically depicts an agent and a phonological pole consisting of a string of sounds ending in /a/ or /or/, depending on the variety of Eng-

\footnotetext{
${ }^{1}$ This is a simplification. The semantic pole of an expression also includes details which are traditionally regarded as pragmatic, such as the fact that trains may not be punctual or that train fares may be relatively high in certain countries. Similarly, the phonological pole should be understood so as to encompass also suprasegmental features like intonation as well as gesture.

${ }^{2}$ Square brackets indicate structures that have unit status, which means that they can be retrieved from memory without much effort, in automatic fashion.
} 
lish considered. In a sense, bound morphemes are partially filled nouns. Similarly, the transitive construction (e.g. Alice shut the door) usually depicts some energetic interaction between two participants, which thus constitutes the expression's semantic pole, and specifies that the word order is Subject Verb Object, at least in statements. $^{3}$

Readers not familiar with the CG framework should thus be able to see that CG is quite radical in that it takes issue with syntax as an "independent" level (or module) of the linguistic system because all syntactic constructions are viewed as assemblies, i.e. combinations, of symbolic structures such as the assembly of the two nominals and a verb in the transitive construction.

Contemporary linguistics does not only usually parcel the linguistic system into discrete components (morphology, syntax, phonology, semantics, etc.) possibly linked by means of "interfaces" but also opts for a formal characterisation of word classes in the sense that word classes are identified on the basis of morphosyntactic criteria (e.g. verbs inflect for tense, nouns do so for number, etc.). Instead, CG, like other "functional" approaches (see e.g. Croft 2001 and Section 3.1 below), also departs from mainstream linguistics because it insists that a conceptual characterisation of word classes is possible, although this does not imply that word classes are necessarily the same across different languages as the conceptual configurations that define their semantic poles may not be symbolised by specific phonological poles in all languages (e.g. not all languages may have a "dedicated" adjective class). Nevertheless, two word classes, namely nouns and verbs, are regarded as probably universal for reasons that will become clearer from the discussion below.

The handling of word classes in $\mathrm{CG}$ is best discussed with reference to Figure 1 (after Langacker 1987: 249), which shows in hierarchical fashion the relations between the semantic poles of various word classes. ${ }^{4}$ In fact, only the shaded boxes in Figure 1 correspond to the semantic poles of actual word classes, the unshaded boxes representing the commonalities between different word classes at a higher level of abstraction.

\footnotetext{
${ }^{3}$ Strictly speaking, word order, which pertains to the phonological pole, cannot be described by using the labels Subject, Verb, Object because these labels refer to bipolar organisation, and so a more complicated description would be needed in CG to represent word order in syntactic constructions. However, for the sake of simplicity and custom, I have used here the conventional labels Subject, Verb, Object to do so. The crucial point is that the reader should bear in mind that these labels are not used in a technical, i.e. CG, sense in the text above.

${ }^{4}$ The focus here will be on the semantic pole of word classes, see instead Hollmann (2012) and (2013) for some discussion of the phonological pole of word classes.
} 


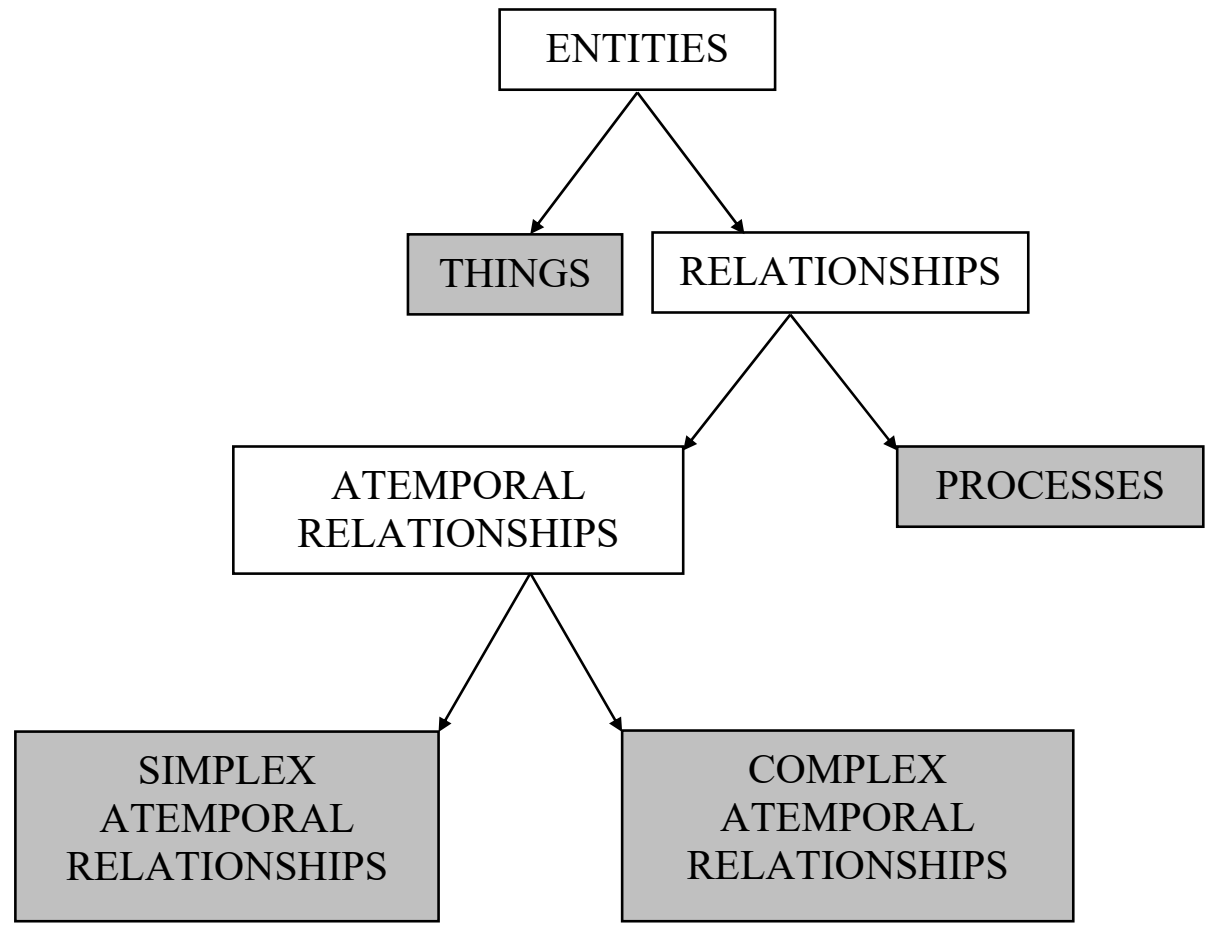

Figure 1. The Cognitive Grammar analysis of (the semantic poles of) word classes

The category ENTITY ${ }^{5}$ includes any type of conception (see Langacker 1987: 198) and subsumes both THING and RELATION (or RELATIONSHIP). The former corresponds to the semantic pole of nouns. Langacker (1987: 198) defines a THING as "a region in some domain", while in more recent formulations, which aim to avoid the spatial metaphor implied by the term "region", Langacker proposes that a THING is the result of the two basic cognitive abilities called grouping and reification (see Langacker 2008a: 105).

For example, on inspecting the dots in Figure 2, we are likely to identify two groups of dots based on colour, namely a group of black dots and a group of white dots. Within the former group, we may identify two subgroups of three dots each, one on the left and one on the right, based on spatial proximity. Further, we may reify the three dots in each black subgroup into a line so that the two subgroups of black dots are construed as two parallel lines.

\footnotetext{
${ }^{5}$ Semantic poles, like phonological poles, should be placed between square brackets (so [ENTITY] rather than ENTITY). Still, for the sake of simplicity, I have refrained from using square brackets here as well as in the rest of the paper.
} 


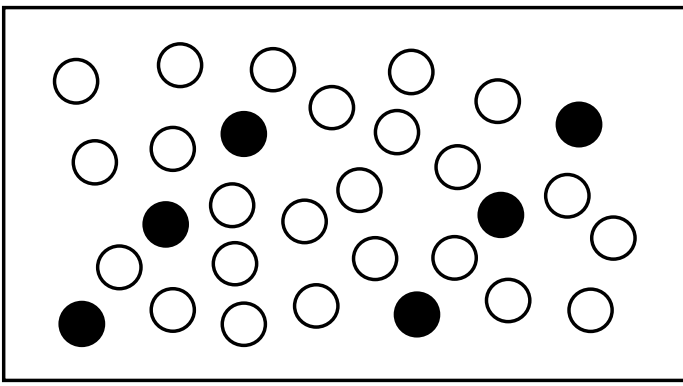

Figure 2. Grouping

a.

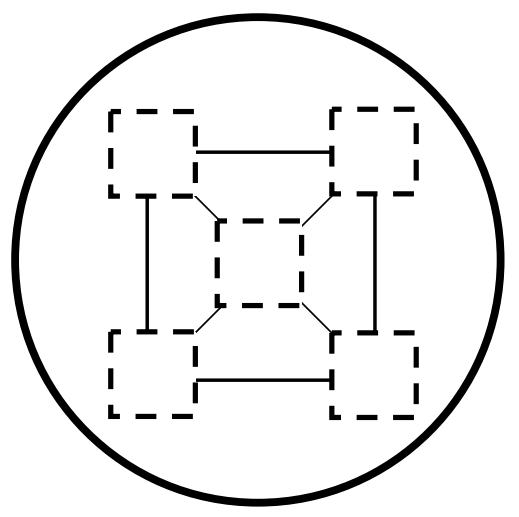

b.

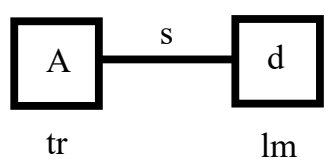

Figure 3. Things (a) and relations (b)

Langacker suggests that we also do the same in the case of nouns or, more generally, nominals (i.e. expressions larger than a single noun such as the kitchen door). The noun team, for example, profiles (i.e. designates) a group of individuals who are reified into a single entity because they function as a unit by sharing a common goal, e.g. that of winning a match, as in Our team won the match comfortably. A usual visual representation for THING is shown in Figure 3a (after Langacker 1987: 215). The diagram makes explicit that what is profiled, as is shown by the use of the circle with a heavy contour, is a set of elements, rather than the elements themselves. These may have some salience but are not as salient as the overall entity, hence the use of dashed heavy lines for the constitutive elements of a THING in Figure 3a. Note also that the connections or relations between the constitutive entities are not profiled either. 


\section{Cristiano Broccias: \\ A new look at word classes in Cognitive Grammar}

While a THING profiles a set of interconnected entities, a RELATION(SHIP) profiles the interconnections between the entities as well as the entities themselves, as is shown in Figure 3b (after Langacker 1987: 215). For example, the verb shut in Alice shut the door describes a relationship (a process, to be exact; see below) because it profiles the interconnection between Alice and the door. Importantly, while we can conceptualise Alice and the door independently of the relation in which they participate, the relation profiled by shut cannot be thought of independently of two participants, Alice and the door in the example at hand. This asymmetry between things and relations is captured in CG by saying that things (and hence nouns) are conceptually autonomous while relations (and hence verbs, for example) are conceptually dependent. Further, within the relation profiled by shut, the status of the two participants, Alice and the door, is different because the former is an "energy source" while the latter is an "energy sink". Thus, CG describes Alice as the trajector ("tr" in Figure 3b) or, technically, the primary focal element of the relation and the door as the landmark ("Im" in Figure 3b) or, technically, the secondary focal element of the relation.

A fundamental distinction is drawn between two types of relationship ${ }^{6}$ : processes and atemporal relations. Langacker (see e.g. Langacker 1987) claims that they are differentiated by virtue of our ability for scanning entities either sequentially (i.e. sequential scanning) or summarily (i.e. summary scanning). Crucially, sequential scanning is taken to be only operative in processes; all other types of relationship are scanned summarily and are collectively referred to as atemporal or nonprocessual relationships. The classic example illustrating the difference between the two scanning modes is the motion of a falling ball (see e.g. Langacker 1987: 144-145). Figure 4 shows successive component states or time slices of the event of a ball falling vertically. Sequential scanning, see Figure 4a, obtains when, tracking the motion of the ball, the conceptualiser focuses on only one component state at a time in her memory through processing time; as time passes, previous component states fade away from her focus of awareness. By contrast, summary scanning, see Figure $4 b$, is "additive" in the sense that the conceptualiser keeps multiple component states active in her working memory through processing time.

\footnotetext{
${ }^{6}$ For the sake of typographical simplicity, from now on I will avoid using capitals for the semantic poles of linguistic expressions. The fact that semantic pole are discussed should be obvious from the text.
} 


\section{EZIKOSLOVLJE}

a.

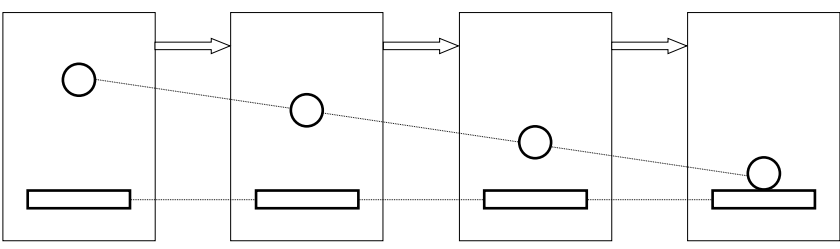

b.

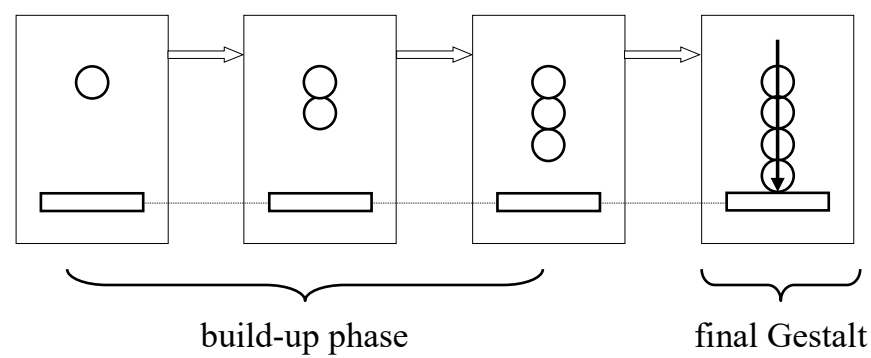

Figure 4. Sequential (a) vs. summary scanning (b) (after Langacker 1987: 144)

The difference is analogous to that between a single exposure photo (sequential scanning) and a multiple exposure photo (summary scanning) of a ball falling. In the latter, we can also reify the various positions occupied by the ball into a higherlevel entity, in analogous fashion to the perception of the two lines in Figure 2 above. This higher-level entity is what we refer to by the nouns trajectory or path, which is shown by means of downward pointing arrow in the final Gestalt in Figure $4 \mathrm{~b}$, which results from a build-up phase where multiple time slices are kept salient. Although the difference between the two scanning modes applies to relationships, the discussion of the nouns trajectory or path shows that scanning also pertains to things. However, Langacker argues that things do not involve sequential scanning, even in the case of event nouns such as explosion. The claim is that the operation of reification, which is constitutive for the apprehension of a thing, makes sequential scanning recede into the background so that even event nouns such as explosion involve summary, not sequential, scanning.

As was anticipated above, processes, and hence the semantic poles of verbs (but see below for more details), are relations that are scanned sequentially. Thus, a more perspicuous representation of a process such as Alice shut the door is along the lines of Figure 5, where an emboldened temporal line is employed to show explicitly that sequential scanning is operative or, to put it differently, that the relation has a "positive" temporal profile. 


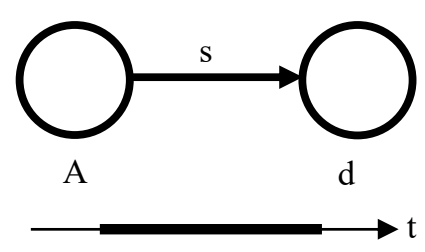

Figure 5. Alice shut the door

a.

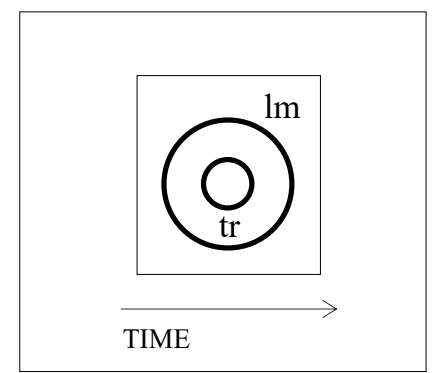

IN

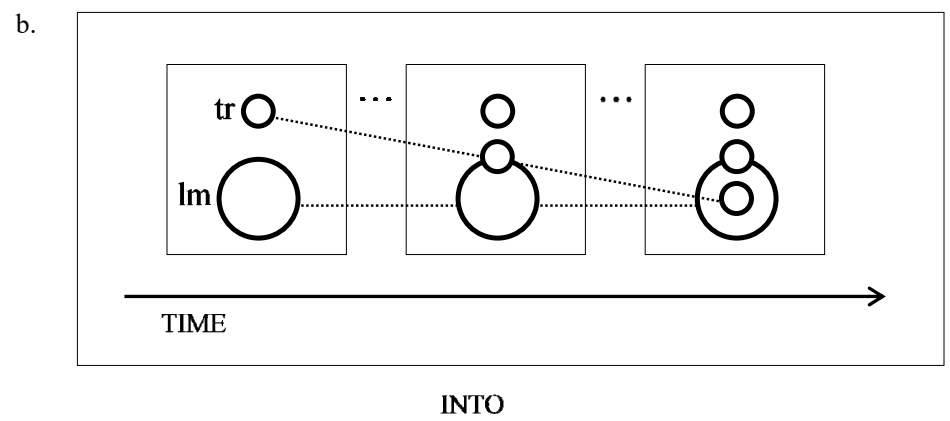

Figure 6. The simplex and complex prepositions in and into

Summarily scanned relations are instead said to have a "null" temporal profile or to be "atemporal", which would be shown by the lack of an emboldened temporal line in diagrams (see e.g. Figure $6 \mathrm{~b}$ above).

Atemporal relations can be either simplex or complex. Simplex atemporal relations are also called stative relations. The difference between simplex and complex 
atemporal relations resides in whether only one component state is active or not. While the simplex preposition in only depicts one component, see Figure 6a, the complex preposition into profiles multiple component states that are scanned summarily, as is shown in Figure 6b. It is instructive to observe already at this juncture that the complex preposition into thus differs from the verb enter because of the scanning mode involved. Both the preposition and the verb have the same content: they depict an entity approaching another entity, with the former ending up being inside the latter. Nevertheless, sequential scanning only applies to the verb enter because it profiles a process, while summary scanning is relevant to the preposition into. Hence, an emboldened temporal line is used only for the verb enter in Figure 7 .

Langacker contends that to-infinitives (e.g. to enter) and participles (e.g. entering, entered) are also complex atemporal relations, which means that they are also scanned summarily (see e.g. Langacker 1991: 200-211). Thus, not all verb forms belong in the same class. While the base form of a verb as well as its tensed forms (e.g. (Alice) enters/entered) are analysed as processes because they are scanned sequentially, the other forms are atemporal relations because they are scanned summarily. Consequently, the overlap between traditional word class labels such as "verb" and the classes shown in Figure 1 above is partial. This is also manifest with prepositions. Dynamic prepositions such as into pattern with non-finite verb forms like entering because they are complex atemporal relations, while stative prepositions like in pattern with adjectives and adverbs in that they are all stative, or simplex, atemporal relations. ${ }^{7}$

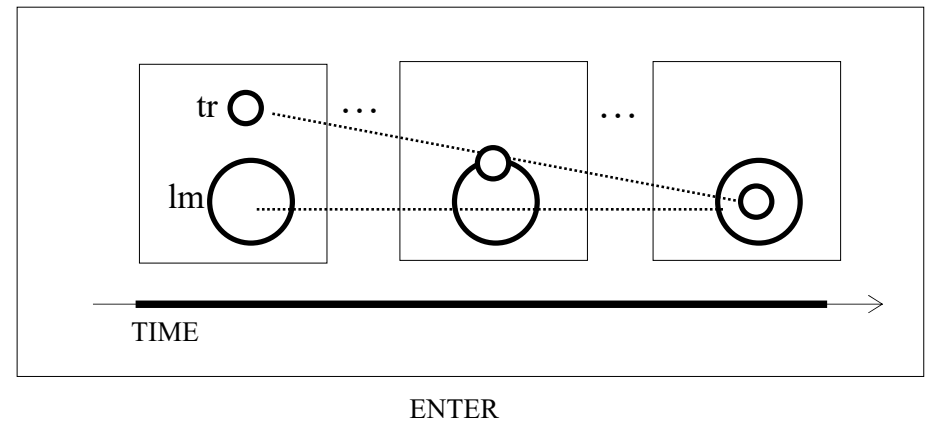

Figure 7. The verb enter

\footnotetext{
${ }^{7}$ Simple atemporal relations are made up of only one component state so that they have a null temporal profile in a trivial sense.
} 
(a) Adjective

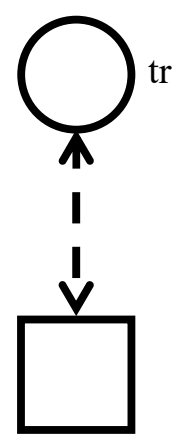

(b) Adverb

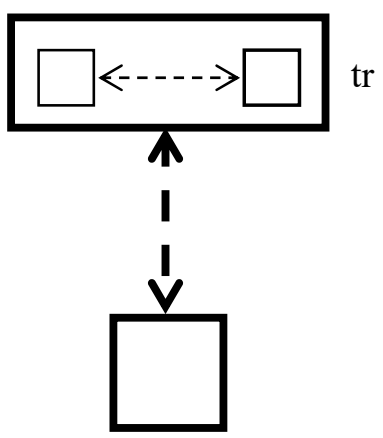

(c) Preposition

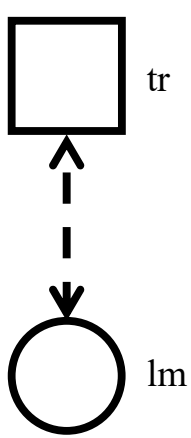

Figure 8. Adjectives, adverbs and prepositions

A diagrammatic representation of adjectives, adverbs and stative prepositions is offered in Figure 8. Adjectives and adverbs differ as to the type of entity that they confer trajector status to. In the case of adjectives (e.g. red), the trajector is a thing (e.g. dress in a red dress) while, in the case of adverbs (e.g. quickly), the trajector is a relation (e.g. read in Alice read the report quickly). In Figure 8a, the circle, as is usual in CG diagrams, see for instance Figure 3a above, stands for a thing (e.g. dress) while the square identifies a region in the colour domain, not shown in the diagram, corresponding to "redness". In Figure 8b, the rectangle identifies a relation (e.g. read), which is shown within it, and the square at the bottom represents a region along a speed scale, not shown in the diagram, corresponding to an abovethe-average speed. In a major departure from earlier work, Langacker (see e.g. Langacker 2008a) has recently argued that not all relations involve a landmark. With regard to adjectives, he explains (Langacker 2008a: 113-114) that

an adjective like pretty, tall, or stupid situates its trajector vis-à-vis a scale representing the degree to which it exhibits a certain property. There is just one focal participant because the adjective itself specifies both the property and the scalar position. Neither is construed as an independently existing entity requiring separate identification.

The same observation of course also extends to adverbs such as quickly. This is why the schematic representation of adjectives and adverbs in Figure 8a-b contains only the label "tr" (trajector) but no label "lm" (landmark). It should also be observed that a landmark may also be lacking with verbs. For example, Langacker contends that a process such as that designated by the motion verb rise (as in The 
waves rose and fell) only involves a trajector but no landmark because the "locations [with respect to which upward motion obtains] are neither individuated nor singled out for separate focus" (Langacker 2008a: 113). Previously, instead, verbs, adjectives and adverbs were all analysed as relationships holding between a trajector and a landmark. Thus, the locations with respect to which the position of the mover (the trajector) is assessed in e.g. rise were analysed as a landmark; also, adjectives, for instance, were said to have an "incorporated" rather than "overt" landmark (see, for example, Taylor 2002: 221, Figure 11.10), corresponding to a region along some property scale. ${ }^{8}$

Unlike adjectives and adverbs, which are relations lacking a landmark, prepositions have both a trajector and a landmark but their trajector can be either a thing or a relation. In the "adjectival" use of, for instance, in as in the woman in the red hat, the trajector is the nominal the woman and the landmark is the nominal the red hat. In the "adverbial" use of in as in She read the report in the cafe, the trajector is the process She read the report and the landmark is the café. The square in Figure 8c stands for an entity, which, as we have seen in Figure 1, can be either a thing or a relation. Although the landmark is shown as a circle in Figure 7c, and hence corresponds to a thing, it must be emphasised that certain prepositions (before, after, un$t i l$, etc.) can also be used as what are traditionally called "subordinating conjunctions" (Bob waited until Alice came back). This happens when the landmark is a relation (Alice came back) rather than a thing. It is a matter of convention whether to call such a use prepositional or not (see e.g. Huddleston \& Pullum 2001 and Aarts 2011 for some discussion). What matters in CG is to elucidate the cognitive abilities and models underpinning how words are used.

\section{Issues with the traditional account}

In this section, I will discuss two issues with the traditional CG account, namely the relevance of the difference between sequential and summary scanning to word classification, which has already been investigated by Broccias and Hollmann (2007), and the notion of relationship, whose exact nature in CG turns out to be somewhat elusive.

\footnotetext{
${ }^{8}$ Although Langacker's characterisation of what constitutes a landmark has changed over time, this does not affect the notion of relationship per se: the point is simply whether all interconnected entities are focussed or not.
} 


\subsection{Scanning modes}

There is no doubt that the two scanning modes have some linguistic import. Nouns such as path, trail and the like clearly offer a summative description of motion, which is consistent with summary scanning. Similarly, the noun trajectory describes the expanse of the infinite number of positions occupied by a body as it moves through space. Still, in keeping with Broccias and Hollmann (2007), we cannot conclude that such "path"-nouns a priori exclude sequential scanning on the part of the language user (see Matlock 2004 for some evidence that they in fact do).

Event nouns may also be problematic when it comes to the distinction between the two scanning modes. Consider the pair in (1), see also Broccias and Hollmann (2007: 497) for a similar point:

\section{(1) a. The building collapsed this morning. \\ b. The collapse of the building took place this morning.}

At least on an intuitive level, it is difficult to see why the verb collapse would involve sequential scanning in (1a) but the event noun collapse would involve summary scanning in (1b), see also Höche (2009). Langacker seems to suggest that reification, as in the case of collapse, necessarily goes hand in hand with summary scanning or, at least, the receding of sequential scanning into the background. This cannot be excluded, of course, but, and this is the crucial point, to lay the foundations for word classes in terms of the two scanning modes is potentially dangerous.

It is also important to distinguish between how scanning operates at the level of a single word and how it operates at the level of more complex phrases. Let us consider, for example, the issue arising from the CG analysis, shown in a compact form in (2b), of the verbal group have been being followed in (2a), see Langacker (1991: 199) ${ }^{9}$ and Broccias and Hollmann (2007).

(2) a. I may very well have been being followed.

b. (have $\left(\mathrm{PERF}_{4}\left(\right.\right.$ be $_{1}\left(-\right.$ ing $\left(\right.$ be $\left.\left.\left.\left._{2}\left(\mathrm{PERF}_{3}(\mathrm{~V})\right)\right)\right)\right)\right)$ )

As was pointed out in Section 2, in CG, bare infinitives involve sequential scanning, while perfects and progressives suspend it so that the resulting relations are said to be scanned summarily. This implies that a series of "scanning oscillations" takes place in the verbal group in (2a): the bare infinitive follow (V in (2b)) would be scanned sequentially, the perfect followed, obtained by combing $\mathrm{V}$ with a perfect marker (shown as $\mathrm{PERF}_{3}$ in (2b)), would be scanned summarily, the infinitive be followed, obtained by combining be (given as be in (2b)) with followed, would

\footnotetext{
${ }^{9}$ The indexes in (1b) are of no particular importance for the rest of the discussion.
} 
be scanned sequentially, the progressive being followed, obtained by combing the bound morpheme -ing with be followed, would be scanned summarily and so on. Obviously, it is natural to ask what the analysis in (2b) is meant to capture: is this how the speaker builds the utterance in (2a) and/or does it capture processing on the part of the listener? More generally, is it cognitively plausible? CG itself insists that much in language is ready-made rather than being constructed piecemeal on each occasion of use, so that it is not clear how the analysis in (2b) would fit in an usage-based approach like CG because the use of the progressive, perfect, and passive constructions should be a relatively automatic task for a proficient user of English. Interestingly, Langacker (2008b: 578) seems to concede that the only scanning mode operative in (2a) is the sequential one because the clause in (2a) is "grounded", i.e. it is related to the speech time, as is evidenced by the modal may. In a similar vein, Langacker (2008b), see also Langacker (2008a: 111), contends that the two scanning modes "should not be thought of as mutually exclusive". These two observations may, at first sight, seem to solve or, at least, alleviate the problem. But, in fact, they complicate the issue further because the two scanning modes are originally invoked to distinguish between word classes (cf. the discussion of into vs. enter above) so that, if we renege on a sharp distinction between the two and/or take grounding as a contributing/deciding factor, then the differences between the various word classes may evaporate. Indeed, Langacker's suggestion concerning the relevance of grounding to scanning may lead us to conclude that, after all, scanning is not necessarily a property of words per se. These are all interesting possibilities, which should ultimately be verified experimentally. Unfortunately, evidence of this kind confirming the relevance of the two scanning modes to word classification in Langacker's sense is lacking (see Kalyan 2016 for an attempt).

Thus, in this paper I aim to explore the possibility of identifying word classes within a CG framework where (a) the difference between the two scanning modes is not crucial for word class identification and (b) sequential scanning is taken to be the default mode of temporal scanning, both at the level of single words and at the level of larger units, whether they be finite or non-finite. In my view, it is especially contentious to claim that bare verbs (e.g. enter), on the one hand, and dynamic prepositions (e.g. into) as well as participles (entering, entered) and to-infinitives (to enter), on the other, differ vis-à-vis scanning. Clearly, the difference between the two types (also?) involves the fact that only the former can be inflected for tense; in other words, only (bare) verbs can be related to the time of speaking, which Langacker views as an instance of grounding. Building on this observation, I will say that verbs (in general, not only bare forms), unlike prepositions, can be 


\section{Cristiano Broccias: \\ A new look at word classes in Cognitive Grammar}

"anchored" to the speech event. Thus, the difference between the finite verb remember and the participial verb locking in

\section{(3) I remember locking the door.}

is, under this view, not (necessarily) one of sequential scanning (remember) vs. summary scanning (locking) as in Langacker's approach, but one of anchoring (remember) vs. lack of anchoring (locking) to the speech event. The fact that grounding or anchoring, as I have called it, is not a cognitive ability but rather a semantic function (see Langacker 2008a: 272) obviously implies that the word classification system that I am proposing is not purely conceptual (see also Diessel 2020), unlike Langacker's. Still, I do not see this as problematic even within a CG framework because there is no compelling theoretical reason why a purely conceptual classification should be preferred over a mixed one which takes into account basic conceptual abilities such as temporal scanning as well semantic functions such as anchoring. Conceptual abilities such as reification may be useful to outline the semantic poles of word classes in very broad terms, e.g. nouns vs. processes, but additional "ingredients" may be necessary for finer-grained distinctions. For example, the conceptual characterisation of various words requires invoking the ground anyway. Just to give an example, the distal determiner this, as in this cat, is based on a relation between the profiled entity (the cat) and the speaker, who is an element of the ground.

In sum, I remain agnostic about the import of the difference between sequential and summary scanning to word classification, which amounts to discounting the two scanning modes as foundational for word class identification. To reiterate, I am not claiming that the two scanning modes are irrelevant tout court in linguistic analysis but that they are problematic as a discriminating factor when it comes to distinguishing between different types of verb forms (finite and bare forms vs. participial and perfect forms), between (finite and bare) verbs and dynamic prepositions, and even, possibly, between (event) nouns and (finite and bare) verbs. Instead, I propose a mixed approach, where cognitive operations as well as semantic functions like anchoring are appealed to in order to identify word classes (see also Croft 2001 for a similar approach).

\subsection{Relations}

In its non-technical (i.e. non-CG) use, the term "relationship" clearly evokes the idea of entities being interconnected to one another. In fact, this also appears to be the way that the term is understood in CG, see Section 2. I have also pointed out 
that a relation does not need to involve two participants; the verb rise was a case in point. It is however important to test the applicability of the notion of relationship against more verbs that, like rise, can be used, in traditional parlance, intransitively. Clearly, cases such as Alice is reading are not problematic because, although not made explicit in the utterance, a relationship obtains between Alice and something which is being read (e.g. a book). Indeed, verbs such as read are usually described as being optionally transitive, which means, in CG terminology, that a landmark is optional. As Langacker correctly points out, an entity involved in a relationship does not necessarily have to be accorded focal prominence, which is precisely what we observe in the example at hand. Nor are particularly problematic cases such as Alice is laughing/crying/shouting, where intransitive verbs of "emission" (see e.g. Broccias 2003) are used. We could argue that a relationship obtains between Alice (the trajector and only focal participant) and an "emitted substance", namely laughs, tears and shouts. Similarly, in Alice is growing very fast, it could be claimed that the change-of-state verb grow depicts a "reflexive" relationship in the sense that Alice is being compared to herself at different stages in her development to conclude that she has grown fast in height, for example. Nevertheless, I contend that not all verbs can easily be described as relationships. Consider, for instance, Alice is sleeping: does sleep really profile a relationship? It is not immediately obvious what this relationship might be. Taylor (2002) is aware of this issue and, in his short discussion of activities (of which sleep is an example) and change-of-state verbs (of which grow is an example), he remarks that, in such instances, the relationship obtains between "the $\operatorname{tr}$ [ajector] and an activity or change-of-state in which the tr[ajector] participates" (see Taylor 2002: 215). In a similar vein, Croft (2001), who uses the notion of "relationality" to distinguish objects, on the one hand, from properties and actions, on the other, writes that relationality involves

whether a definition of a concept inherently requires reference to another concept (Langacker 1987: 214-16). For example, one cannot conceive of an action such as running without the involvement of a runner, or of a property such as height without something that is tall. On the other hand, one can conceive of a chair or a dog without the involvement of another concept.

I believe that both Taylor's and Croft's characterisations do not actually correspond to Langacker's notion of relationship. Taylor seems to suggest that, if we use the examples above for illustrative purposes, then a relationship obtains between Alice and the activity of sleeping in Alice is sleeping and between Alice and the change of state designated by grow in Alice is growing very fast. This formulation, however, is not very clear because activities and changes of state, by being processes, are not conceptually autonomous (see Section 2 ) but are dependent. They necessarily 


\section{Cristiano Broccias: \\ A new look at word classes in Cognitive Grammar}

involve some entities so that what Taylor seems to refer to is the traditional notion of "predication": what we are predicating of Alice is that she is sleeping and that she is growing fast. But this is an additional relation obtaining between the putative relation itself (i.e. sleep/grow) and its participant (Alice); it is not a relation between entities "within" (i.e. connected by) the profiled relation. I will refer to the "predicative" use of the term relation as "external" while I will refer to the use of the term "relation" in Langacker's sense as "internal". The difference between the two cases is captured in Figure 9a for the transitive example Alice likes cats and in Figure $9 \mathrm{~b}$ for the intransitive example Alice is sleeping (for the sake of simplicity the time arrow has been omitted). The question mark in Figure 9b shows that it is not clear what constitutes the "internal" relation in (some) intransitive examples.

Croft (2001) also uses "relationality" differently from Langacker's notion of relation. In the passage quoted above, he seems to be describing the distinction between autonomous and dependent concepts. But the term "relationship" is not used in CG to just denote the dependency of a concept on its participants but rather to denote the interconnections between the entities involved (see Langacker 2008a: 113) so that the action of running used by Croft as an illustrative example is of course relational in the sense of being dependent but is not necessarily relational in the (internal) sense of depicting a relation between the runner and someone or something else.

In sum, in both Alice likes cats and Alice is sleeping, we can claim that an "external" relationship obtains between the trajector (Alice) and some "process", independently of its exact nature. This is simply a matter of predication, traditionally understood. It does not mean that the nature of the processes like and sleep is relational in both cases. Of course, we could try to offer an "internal" relationship analysis of cases such as Alice is sleeping by claiming that there exists a relationship between a trajector and a state (cf. Alice is asleep), provided that the latter is not itself conceptualised as a relationship, so as not to confuse again an external relationship with an internal one. The possibility of using sleep in the cognate object construction (see Levin 1993) as in Alice is sleeping a sound sleep provides some ground for a relational characterisation of the internal type, that is a relation between Alice and sleep. Still, this usage is marginal compared to the intransitive one and a relational analysis is less obvious than, for example, with "emission" verbs such as laugh, which are also intransitive and can also appear in the cognate object construction (e.g. Alice is laughing a silly laugh) but clearly describe a relation between an emitter and an emitted substance (e.g. laughs). 


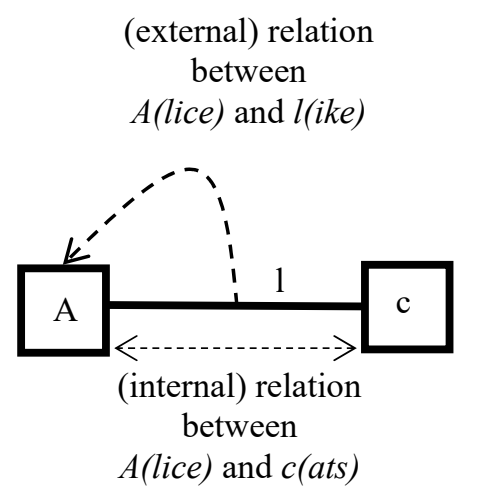

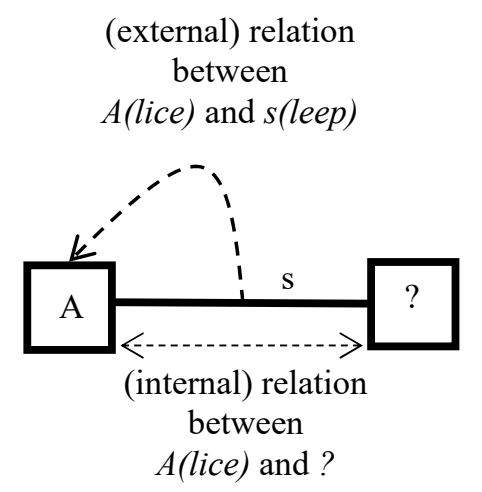

Figure 9. Internal and external relations

Thus, in order not to impose a necessary (internally) relational conceptualisation on verbs such as sleep, I will dispense of the notion of (internal) relationship as critical for separating nouns from other word classes.

\section{An alternative CG account}

In Langacker's framework, the identification of word classes fundamentally rests on two differences: whether an entity is relational or non-relational and whether a relationship is scanned sequentially or summarily. Nevertheless, as was pointed out above, the relevance of the two scanning modes to word classification is not unproblematic and the notion of relationship may not be easy to reconcile with all types of verbs. I have contended that it is safer to opt for a mixed approach, where basic cognitive operations are not sufficient for identifying word classes but are supplemented with other criteria. For example, if we assume that both enter and into depict relationships scanned sequentially, they can still be distinguished by appealing to anchoring. The preposition into cannot be anchored to the speech event, which in turn means that it is dependent on another element, whether it be explicit (e.g. the verb went in Alice went into the room) or implicit (e.g. Where did Alice go? Into the room, where into the room is dependent on go although it is absent in the answer).

If we reject the relational description of non-nominal word classes, at least as used by Langacker, the obvious question is how we can distinguish between what Langacker calls nouns from his class of relations. 


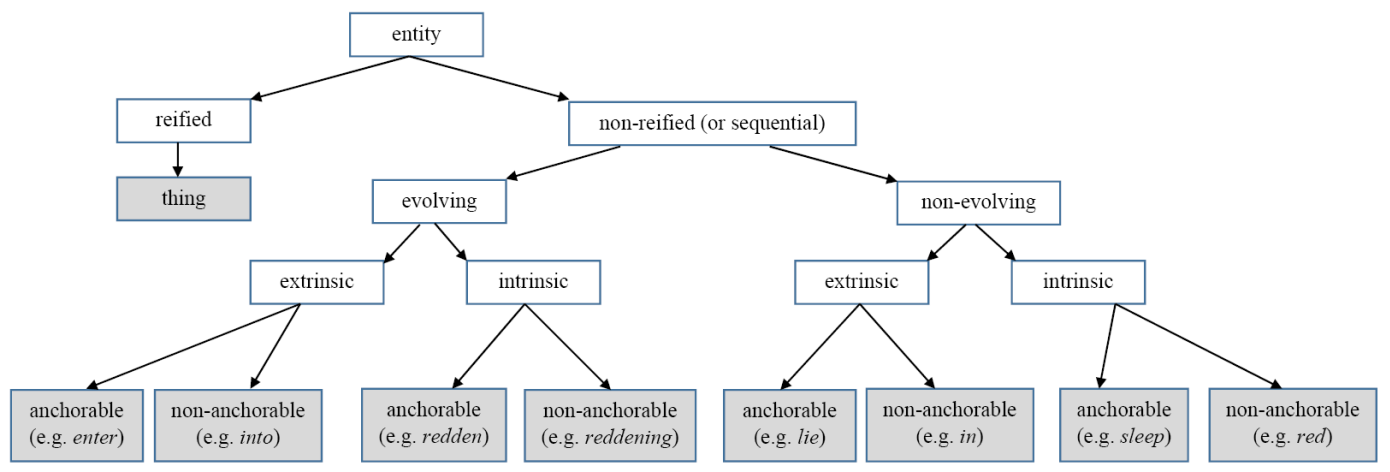

Figure 10. An alternative view of word classes in CG

I think that we can still do so by relying on our ability for reification and temporal scanning but in a different way from Langacker's. This is illustrated in a compact way in Figure 10, which is useful to refer to in the rest of the discussion.

We can separate the class of nouns from the other classes by noticing that only the former involves reification. Thus, I contend that the basic distinction is between reification and lack of reification, which is in line with Langacker's approach. Still, I believe that it is safer to remain agnostic about whether reification necessarily implies summary scanning (see e.g. the short discussion of explosion in the previous section). It is clear that actions can be treated as objects that, for example, can be transferred (e.g. nod in Alice gave Bob a nod) but I leave to future research a more precise characterisation of reification. We cannot exclude, for instance, that the reification of actions is the result of blending, in Fauconnier and Turner's (2002) sense, of an action and a prototypical physical object. Thus, the sequential scanning implied by the root verb (e.g. explode, nod) could be projected into the blend (the event noun explosion and nod), without the need for sequential scanning to be suspended, unlike in Langacker's approach. Still, my focus here is on Langacker's relations. Although I suggest viewing them as non-reified entities, I think that more can be said about them if, as in Langacker's approach, we focus on the issue of scanning. Let us consider Alice entered the room, first, see Figure 11a. The conceptualiser (i.e. the language user) must track the position of the trajector (Alice) with respect to the landmark (the room) ${ }^{10}$ through time and detect a change in their relation.

\footnotetext{
${ }^{10}$ For the sake of expository convenience, I am analysing the room as a landmark although Langacker (2008a) would probably object to it.
} 
a.

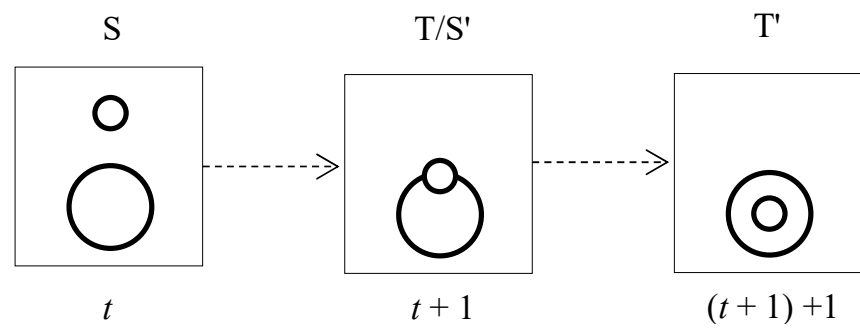

b.

$\mathrm{S}$

$\mathrm{T} / \mathrm{S}^{\prime}$

$\mathrm{T}^{\prime}$

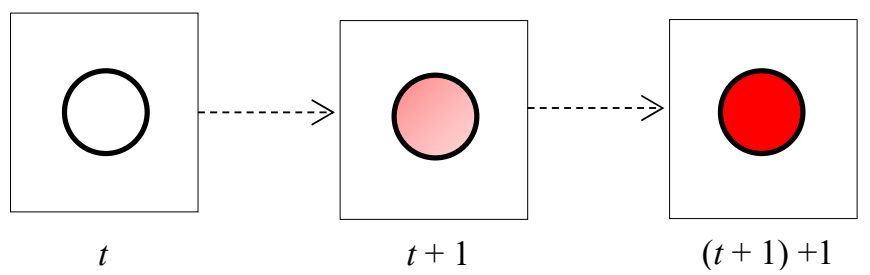

Figure 11. Evolving categorization sequences

This is done by comparing each component state or time slice (i.e. the position of Alice vis-à-vis the room at a certain point in time) with the following one, thus creating a sequence of operations of categorisation. (For the sake of simplicity, Figure 11 reduces the process of entering down to three time slices.) In CG terminology, categorisation resides in the operation of comparison between a standard (S) of categorisation and a target $(\mathrm{T})$ of categorisation. Here, a component state at time $t$, which can metaphorically speaking be thought of as a photo taken at time $t$, is the standard for the component state at time $t+1$, which then functions as the target of categorisation. This target is then used as a new standard ( $\left.\mathrm{S}^{\prime}\right)$ for a new target $\left(\mathrm{T}^{\prime}\right)$, which is the next time slice, at time $(t+1)+1$. The conceptualiser detects both similarities and differences between the two component states (Alice and the room are present in both component states but Alice's position relative to the room has changed) that thus define a changing or evolving categorisation sequence, a subtype of what I will refer to as sequential categorisation. This is basically what Langacker would call sequential scanning in the case of change-of-position or change-of-state processes. There is of course a relational element in the creation of an evolving categorisation sequence but this type of relationality is very different from Langacker's (or Croft's, for that matter). The relation that I have in mind obtains between the successive time slices during the act of categorisation performed 
by the conceptualiser. In the case at hand, the relation is one of partial overlap between the standard and target of categorisation or "extension" in CG terminology, which is shown by a dashed arrow in Figure 11: the component states are similar but not identical. This does not detract from the fact that there is also a relation between two entities (Alice and the room), which I think is what Langacker (but not Croft) would label as a "relationship". As two separate entities are involved, I will label the evolving categorisation sequence under discussion "extrinsic".

Let us now consider Alice's face reddened, see Figure 11b for a much simplified representation. The described event is still an evolving categorisation sequence because the time slices are not identical to one another but the change taking place is assessed only with reference to Alice's face (the circle in Figure 10b). As only one entity is involved, I will label such an evolving categorisation sequence "intrinsic". In a sense, the term intrinsic sequence could also be interpreted as subsuming what Taylor views as a relation with an "incorporated landmark", although Langacker's more recent formulation discounts the existence of such landmarks. To put it differently, Alice's face reddened could also be analysed as a relation between Alice's face (the trajector) and a region (the landmark) in the colour domain, which is identified by the verb itself as being (eventually) red.

We can now tackle Alice slept for hours, which unlike the previous examples depicts an activity in Vendler's (1967) terminology, and contains the verb sleep for which a(n internal) relational analysis may not be obvious or even appropriate (see the previous Section). Although sleeping may involve change, for example Alice's position in the bed, Alice starting or stopping snoring, etc., the various component states are probably construed as being identical at a suitable level of granularity. In other words, this activity can be treated as an instance of a non-evolving categorisation sequence in the sense that the standard and the target of comparison are construed as being identical, see Figure 12, which shows, by means of continuous arrows rather than dashed ones connecting the various component states, that each target is an instantiation of the standard rather than an extension, as is the case with change-of-position/state processes. Further, as the activity only concerns Alice, the sequence under discussion can be described as intrinsic. Under the reading of "intrinsic" as designating the property of having an incorporated landmark, we could conceive of sleeping as a relationship between Alice and the state of being asleep, although I have refrained from describing all events as relations. Still, the use of the label "intrinsic" leaves the door open for such a characterisation if it is proven to be viable. 


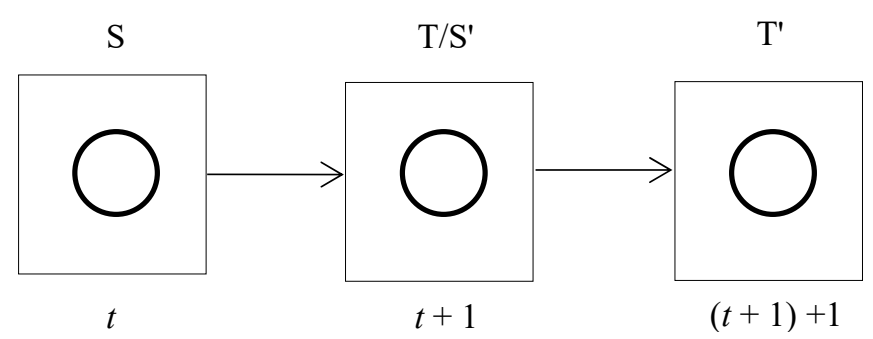

Figure 12. Non-evolving categorization sequences

Importantly, I have observed that lack of evolution in a categorisation sequence is a matter of construal; the component states of the activity sleep are construed as being identical at an appropriate level of granularity. But construal does not only pertain to the categorising operation of comparison between successive component states (so that they are deemed to be identical or not) but also to whether the categorisation sequence is intrinsic or extrinsic. For example, if we augmented the example above by means of a locative phrase as in Alice slept on the couch for hours, we could argue that slept describes not only an activity on Alice's part but also a relation between Alice and the couch. It seems that Langacker's more recent formulation of CG would discount the possibility of analysing the couch as a participant in this instance, as this label is only reserved for elements which participate directly in a relationship (see Section 2). Still, it is well-known that this is a matter of construal, as is shown by passive examples such as The bed hasn't been slept in, where passivisation is taken as a diagnostic for "direct participation" in the event. Thus, again, I leave the issue of whether a categorisation sequence is intrinsic or non-extrinsic open to construal. Intuitively, slept, as in Alice slept for hours, is more intrinsic than laid (cf. Alice laid for hours vs. the more obvious option Alice laid on the floor for hours). Hence, for the sake of convenience, I will say that lie as in Alice laid on the floor for hours illustrates an extrinsic categorisation sequence, which, like that for sleep, is not evolving.

I propose that categorisation sequences are also relevant to other non-evolving cases. While Langacker would claim that adjectives such as red and stative prepositions such as in do not have a positive temporal profile (i.e. no sequential scanning is involved) and in this respect behave in the same way as reddening (as in $a$ reddening face) and into, I contend that all four cases (red, reddening, in, into) can be analysed as categorisation sequences and that we distinguish between reddening and into, on the one hand, and red and in, on the other, on the basis of whether the 


\section{Cristiano Broccias: \\ A new look at word classes in Cognitive Grammar}

categorisation sequence is evolving (reddening and into) or not (red and in). ${ }^{11}$ Cross-cutting this difference is that between sequences that are extrinsic vs. sequences that are intrinsic. Prepositions clearly portray extrinsic relations while adjectives do not always do so. ${ }^{12}$ Nevertheless, even if we appeal to reification and temporality in the characterisation of word classes, a purely conceptual description appears to be elusive. This of course is a consequence of rejecting the importance of the contrast between summary and sequential scanning, if it exists at all. We need to invoke anchorability to distinguish for example bare verbs such as redden, on the one hand, from participles (reddened, reddening) as well as adjectives (red), on the other, in that only the former can be anchored to the speech event. Similarly, the contrast between the bare verb enter and the dynamic preposition into is also one of anchoring. Only enter can be anchored to the speech event, while into, like the stative preposition in, for that matter, requires (explicit or implicit) dependency on a predicate.

\section{Conclusion}

This paper has shown that pivotal to the CG conceptual characterisation of word classes are the difference between sequential and summary scanning and the notion of relation. Nevertheless, as both are potentially problematic, an attempt has been made to develop a classificatory system for word classes that does not make use of them but is still consistent with CG assumptions. Firstly, it has been proposed that both verbs and "dynamic" prepositions involve sequential scanning but differ, quite simply, as to whether the profiled process can be anchored temporally. Secondly, it has been suggested that Langacker's relations are best analysed as categorisation sequences, which can be divided into two types: evolving (e.g. redden) and nonevolving (e.g. red as an adjective). Thirdly, categorisation sequences have been classified as either extrinsic or intrinsic, depending on whether both a trajector and a landmark are present or only a trajector is involved, respectively. It has been pointed out, however, that the difference between extrinsic and intrinsic sequences

\footnotetext{
${ }^{11}$ This does not mean that all adjectives are non-evolving categorisation sequences. Consider a classic example such as the adjective future in the future president. This adjective can be classified as an intrinsic, evolving categorisation sequence because the speaker engages in a mental simulation whereby she detects a contrast between a present-time component state of reality and a future-time component state of projected reality, in which somebody will play a role that they do not have in current reality.

${ }^{12}$ In Her dress is red, the adjective red depicts an intrinsic sequence but in Alice is tired of her life, there is also an extrinsic relation between Alice and her life.
} 
may be a matter of construal. Although this paper has focussed on Langacker's notion of relationship as well as scanning, future research should investigate the nature of reification in more detail because this cognitive ability may be fundamental for separating nominals from non-nominals. I have suggested in passing that reification may not be incompatible with sequential scanning, unlike in Langacker's approach, if reification is understood in terms of conceptual blending. This is surely a topic that needs further exploration so as to test the feasibility and the limits of a CG approach to word classification.

\section{References}

Aarts, Bas. 2011. Oxford Modern English grammar. Oxford: Oxford University Press.

Broccias, Cristiano. 2003. The English change network: Forcing changes into schemas. Berlin: Mouton de Gruyter. https://doi.org/10.1515/9783110901207

Broccias, Cristiano \& Hollmann, Willem B. 2007. Do we need summary and sequential scanning in (Cognitive) Grammar? Cognitive Linguistics 18. 487-522. https://doi.org/10.1515/COG.2007.026

Croft, William. 2001. Radical Construction Grammar: Syntactic theory in typological perspective. Oxford: Oxford University Press. doi:10.1093/acprof:oso/9780198299554.001.0001

Diessel, Holger. 2020. A dynamic network approach to the study of syntax. Frontiers in Psychology 11. article 604853. https://doi.org/10.3389/fpsyg.2020.604853

Fauconnier, Gilles \& Turner, Mark. 2002. The way we think: Conceptual blending and the mind's hidden complexities. New York: Basic Books.

Höche, Silke. 2009. Cognate object constructions in English: A cognitive-linguistic account. Tübingen: Gunter Narr Verlag Tübingen.

Hollmann, Willem B. 2012. Word classes: Towards a more comprehensive usage-based account. Studies in Language 36. 671-698. https://doi.org/10.1075/sl.36.3.08hol

Hollmann, Willem B. 2013. Nouns and verbs in Cognitive Grammar: Where is the 'sound' evidence? Cognitive Linguistics 24. 275-308. https://doi.org/10.1515/cog-2013-0009

Huddleston, Rodney \& Pullum, Geoffrey K. 2002. The Cambridge grammar of the English Language. Cambridge: Cambridge University Press. https://doi.org/10.1017/9781316423530

Kalyan, Siva. 2016. Operationalising Cognitive Grammar: Experimental and theoretical approaches. Unpublished doctoral dissertation, Newcastle upon Tyne: Northumbria University (UK).

Langacker, Ronald W. 1987. Foundations of Cognitive Grammar, vol. 1, Theoretical prerequisites. Stanford: Stanford University Press. 


\section{Cristiano Broccias: \\ A new look at word classes in Cognitive Grammar}

Langacker, Ronald W. 1990. Concept, image, and symbol: The cognitive basis of grammar. Berlin: Mouton de Gruyter. https://doi.org/10.1515/9783110857733

Langacker, Ronald W. 1991. Foundations of Cognitive Grammar, vol. 2, Descriptive application. Stanford: Stanford University Press.

Langacker, Ronald W. 1999. Grammar and conceptualization. Berlin: Mouton de Gruyter. https://doi.org/10.1515/9783110800524

Langacker Ronald W. 2008a. Cognitive Grammar: A basic introduction. New York: Oxford University Press. doi:10.1093/acprof:oso/9780195331967.001.0001

Langacker, Ronald W. 2008b. Sequential and summary scanning: A reply. Cognitive Linguistics 19. 571-584. https://doi.org/10.1515/COGL.2008.022

Langacker, Ronald W. 2009. Investigations in Cognitive Grammar. Berlin: Mouton de Gruyter. https://doi.org/10.1515/9783110214369

Levin, Beth. 1993. English verb classes and alternations. Chicago, IL: University of Chicago Press.

Matlock, Teenie. 2004. Fictive motion as cognitive simulation. Memory and Cognition 32. 1389-1400. https://doi.org/10.3758/BF03206329

Taylor, John R. 2002. Cognitive Grammar. Oxford: Oxford University Press.

Vendler, Zeno. 1967. Linguistics in philosophy. Ithaca, NY: Cornell University Press. https://doi.org/10.7591/9781501743726

\section{Author's address:}

Dipartimento di Lingue e Culture Moderne

Università di Genova

Piazza S. Sabina 2, 16124 Genova

E-mail: c.broccias@unige.it

\section{EIN NEUER BLICK AUF WORTKLASSEN IN DER KOGNITIVEN GRAMMATIK}

Langackers Kognitive Grammatik zielt darauf ab, eine konzeptionelle Charakterisierung von Wortklassen anzubieten. Zentral für dieses Bestreben ist die Unterscheidung zwischen "Ding" und "Relation" sowie die Behauptung, dass Relationen entweder sequentiell oder zusammenfassend gescannt werden können. Der Unterschied zwischen den beiden Arten des Scannens ist wichtig, um beispielsweise zwischen dem Verb enter und der dynamischen Präposition into differenzieren zu können. In diesem Beitrag wird die Debatte über den Status und die Relevanz der beiden Arten des Scannens neu aufgegriffen und der Begriff der Relation selbst einer genauen Prüfung unterzogen, um seine potenziell problematische Natur zu enthüllen. Es wird eine neue Sichtweise entwickelt, die auf den Begriffen evolvierende vs. nicht-evolvierende Kategorisierungssequenzen, Extrinsikalität vs. 
Intrinsikalität und Verankerung basiert, die eine radikale Abkehr von der Kognitiven Grammatik darstellt, obwohl sie mit ihren Hauptgrundsätzen kompatibel ist.

Schlüsselwörter: Wortklassen; Kognitive Grammatik; zusammenfassendes Scannen; sequentielles Scannen; Relation. 